\title{
QUÍMICA, PEDAGOGÍA Y AUTORIDAD EXPERTA: EL TRATADO DE ANÁLISIS QUÍMICO DE JOSÉ CASARES GIL (1866-1961) Y LA CONSTRUCCIÓN DE UNA DISCIPLINA CIENTÍFICA
}

\author{
Ignacio Suay-Matallana \\ CIUHCT - Centro Interuniversitário de História das Ciências e da Tecnologia \\ i.matallana@fct.unl.pt
}

Recibido: 25 octubre 2015; Aceptado: 17 junio 2016

\begin{abstract}
Cómo citar este artículo/Citation: Suay-Matallana, Ignacio (2016), “Química, pedagogía y autoridad experta: el Tratado de análisis químico de José Casares Gil (1866-1961) y la construcción de una disciplina científica", Asclepio, 68 (2): p154. doi: http://dx.doi. org/10.3989/asclepio.2016.26

RESUMEN: En este artículo se estudiará cómo José Casares utilizó sus libros de texto y su reconocimiento como experto para consolidar el análisis químico como disciplina en las universidades españolas. En primer lugar se mostrará el papel activo de Casares en la configuración de sus libros de texto. Gracias a sus publicaciones, algunos autores de libros de ciencias se convirtieron en voces autorizadas en su disciplina y las promocionaban en sus contextos locales. El estudio del tratado publicado por Casares muestra también los recursos utilizados por su autor para afirmar su disciplina ante públicos diversos. En segundo lugar se describe el origen y la evolución de su tratado, así como sus principales elementos configurativos. Finalmente, se examinan las estrategias utilizadas por Casares para construir una identidad disciplinar en torno a la química analítica en España.
\end{abstract}

PALABRAS CLAVE: Libros de texto; Química; Disciplinas científicas; España; Siglo XX.

CHEMISTRY, PEDAGOGY, AND SCIENTIFIC AUTHORITY: THE TRATADO DE ANÁLISIS QUÍMICO BY JOSÉ CASARES GIL (1866-1961), AND THE SHAPING OF A SCIENTIFIC DISCIPLINE

ABSTRACT: This article will explore how José Casares employed his textbooks and his recognition as expert to consolidate chemical analysis as a discipline in the Spanish universities. First, it will be shown the active role of Casares in the shaping of his textbooks. Thanks to their publications, textbook authors became trusted voices in their disciplines, promoting them in their local contexts. The study of the Casares' treatise also shows the resources employed to establish his discipline among different publics. Secondly, the article will describe the origin and the evolution of his treatise, as well as its main shaping elements. Finally, the article will examine the strategies developed by Casares to construct a disciplinary identity for analytical chemistry in Spain.

KEY WORDS: Textbooks; Chemistry; Scientific disciplines; Spain; 20th century.

Copyright: (C) 2016 CSIC. Este es un artículo de acceso abierto distribuido bajo los términos de la licencia Creative Commons Attribution (CC BY) España 3.0. 


\section{INTRODUCCIÓN: LIBROS DE TEXTO, EXPERTOS Y DISCIPLINAS}

A lo largo de los siglos XIX y XX, la enseñanza de las ciencias sufrió grandes transformaciones que favorecieron la consolidación de un género de literatura científica particularmente diseñado para las aulas y para un nuevo tipo de enseñanza: los libros de texto y los manuales académicos. Hasta hace unas décadas y a pesar de su importancia indudable, los libros de texto raramente habían sido considerados como objetos de estudio con valor propio. Por regla general, han sido utilizados como meras fuentes documentales o escaparates del conocimiento científico establecido y acumulado, dentro de modelos más o menos difusionistas de la circulación del conocimiento. Al igual que ha ocurrido en otros aspectos de la ciencia, este orden jerárquico entre investigación y enseñanza, con sus respectivos roles preestablecidos, ha sido fuertemente cuestionado (Vicedo, 2012; Kaiser, 2005).

Esta renovada aproximación histórica hacia los manuales ha permitido reconsiderar las relaciones existentes entre los integrantes del "circuito del libro», que incluye tanto a autores y públicos destinatarios como a editores especializados, libreros, regulaciones estatales y mercados editoriales (Darnton, 2007). Los autores y los traductores tienen un papel activo en la elaboración de un libro de texto debido a sus decisiones sobre la organización del texto, la selección de los públicos destinatarios, la elección y cooperación con la editorial, y la consideración y adaptación a las regulaciones legales existentes ${ }^{1}$. Por ello, la publicación de libros de texto, tratados y manuales permitió que los científicos definieran los límites disciplinares de las materias en las que trabajaban y fueran identificados como expertos por públicos diversos. Además, la confluencia de estudios procedentes de la historia de la educación y de la historia de la ciencia - muchos de ellos inspirados en los trabajos de Michel Foucault (1926-1984) - han puesto de manifiesto la estrecha relación entre los manuales y la formación de disciplinas académicas. Los libros de texto son un elemento crucial en la definición de los límites de una disciplina y sus conexiones con otras áreas académicas y, además, proporcionan numerosos detalles para conocer la estructura interna de la disciplina y las normas, valores y actitudes éticas que regulan una comunidad académica (Bertomeu, García, Lundgren y Pationitis, 2006).

En este trabajo se estudia el manual de análisis químico publicado por José Casares Gil (1866-1961), que circuló ampliamente en las facultades de química y farmacia, con la intención de mostrar su contribución a la creación de una identidad disciplinar de la química analítica en las universidades españolas de la primera mitad del siglo XX² . La química analítica y el análisis químico han sido consideradas como una de las áreas de la química con más larga tradición. En el libro History of Analytical Chemistry, que publicó el químico e historiador Ferenc Szabadváry (1923-2006), se afirmaba rotundamente que «la química analítica era la madre de la química moderna» (Szabadvary, 1966). Esta obra, también dedicaba un apartado a los primeros libros de química analítica e indicaba que los científicos alemanes fueron quienes lideraron «indudablemente» este área disciplinar hasta la segunda guerra mundial ${ }^{3}$. En el ámbito académico español existían dos tradiciones vinculadas a los análisis químicos distinguiéndose entre los «químicos de farmacia» y los «químicos de ciencias» según procedieran de facultades de farmacia o de ciencias (González, 2005). Sin embargo, además de los químicos y los farmacéuticos - dedicados a los análisis químicos de aguas minerales, alimentos, medicamentos o muestras forenses - también existían otros grupos profesionales dedicados a los análisis químicos, como los médicos, los geólogos o los ingenieros así como los ensayadores de metales que se dedicaban al estudio de rocas, minerales y aleaciones.

Desde mediados del siglo XIX, la pedagogía de la química se esforzó en defender los trabajos experimentales y fomentó la mejora de las enseñanzas prácticas. El desarrollo de este tipo de enseñanza implicó que aparecieran nuevos libros de texto para servir de apoyo a las nuevas prácticas docentes (Bertomeu y García, 2002). En España este esfuerzo modernizador se vio complementado por el impulso renovador de algunos profesores vinculados, principalmente, a la Junta para Ampliación de Estudios e Investigaciones Científicas (JAE), creada en 1907 y presidida por Santiago Ramón y Cajal (1852-1934). Las principales iniciativas de la JAE consistieron en el impulso de los viajes científicos, la mejora de las enseñanzas prácticas de futuros maestros, profesores y catedráticos, el impulso de las prácticas experimentales y la promoción de las actividades divulgadoras de la ciencia (Barona, 2007). José Casares fue miembro de la JAE desde su fundación y participó de las actividades institucionales relacionadas con la educación en el exterior con las que se pretendía regenerar la ciencia española.

Numerosos historiadores españoles han señalado que «la auténtica importancia de José Casares se centra en su labor pedagógica» ya que «su labor como publicista fue muy copiosa y de importancia» (Portela, 1983; Roldán, 1975). Efectivamente, José 
Casares ejerció como catedrático de análisis químico entre 1888 y 1936 en las facultades de farmacia de Barcelona y Madrid, en las que también llegó a ser decano. Casares fue autor de numerosos manuales y tratados, algunos de los cuales circularon ampliamente por las aulas españolas, como por ejemplo el Tratado de análisis químico (publicado entre 1897 y 1978) estudiado en este trabajo o el Tratado de técnica física (que gracias a sus cuatro ediciones publicadas entre 1908 y 1932, contribuyó a renovar las prácticas experimentales de la química española en el primer tercio del siglo XX). Además, Casares fue director del laboratorio central de aduanas (1908-1951) y gozó de un relevante peso institucional tanto durante la monarquía, como durante el periodo republicano y el franquista, ya que fue nombrado senador, miembro de la Junta para Ampliación de Estudios e Investigaciones Científicas (JAE), procurador en cortes y director de las Reales Academias de Farmacia y de Ciencias Exactas, Físicas y Naturales (Suay-Matallana, 2014a). Es decir, José Casares fue considerado tanto un reconocido profesor y autor de manuales científicos como un destacado experto químico con gran peso en la esfera pública de su época. Por ello, es muy relevante el estudio de su papel en la creación de la identidad disciplinar de una nueva especialidad emergente como era la química analítica.

En los apartados siguientes se estudiará el Tratado de análisis químico, el texto más reconocido de Casares y que, a lo largo de sus trece ediciones publicadas entre 1897 y 1978 contribuyó, decisivamente a la consolidación de la química analítica en las aulas españolas (figura 1). A continuación se examinarán algunos de los rasgos más característicos de las múltiples ediciones que tuvo su Tratado de análisis químico, así como la evolución de la obra y los esfuerzos del autor para organizar y adaptar las sucesivas ediciones. Finalmente, se analizará el modo en el que este tratado contribuyó a expandir la química analítica en la sociedad española de principios del siglo XX, al tiempo que ayudó a que José Casares fuera considerado como el principal experto en esta materia.

\section{ORIGEN, EVOLUCIÓN Y ORGANIZACIÓN DE UN TRATADO ESPECIALIZADO}

Los libros de texto están, en numerosas ocasiones, estrechamente vinculados a los viajes de estudio al extranjero y el interés de los profesores por adaptar las experiencias allí aprendidas a sus clases (García y Bertomeu, 2001). En el caso de Casares, uno de los
Figura 1. Portadas de diferentes ediciones del libro de análisis químico publicado por José Casares. En 1897, 1905, 1911 y 1918.

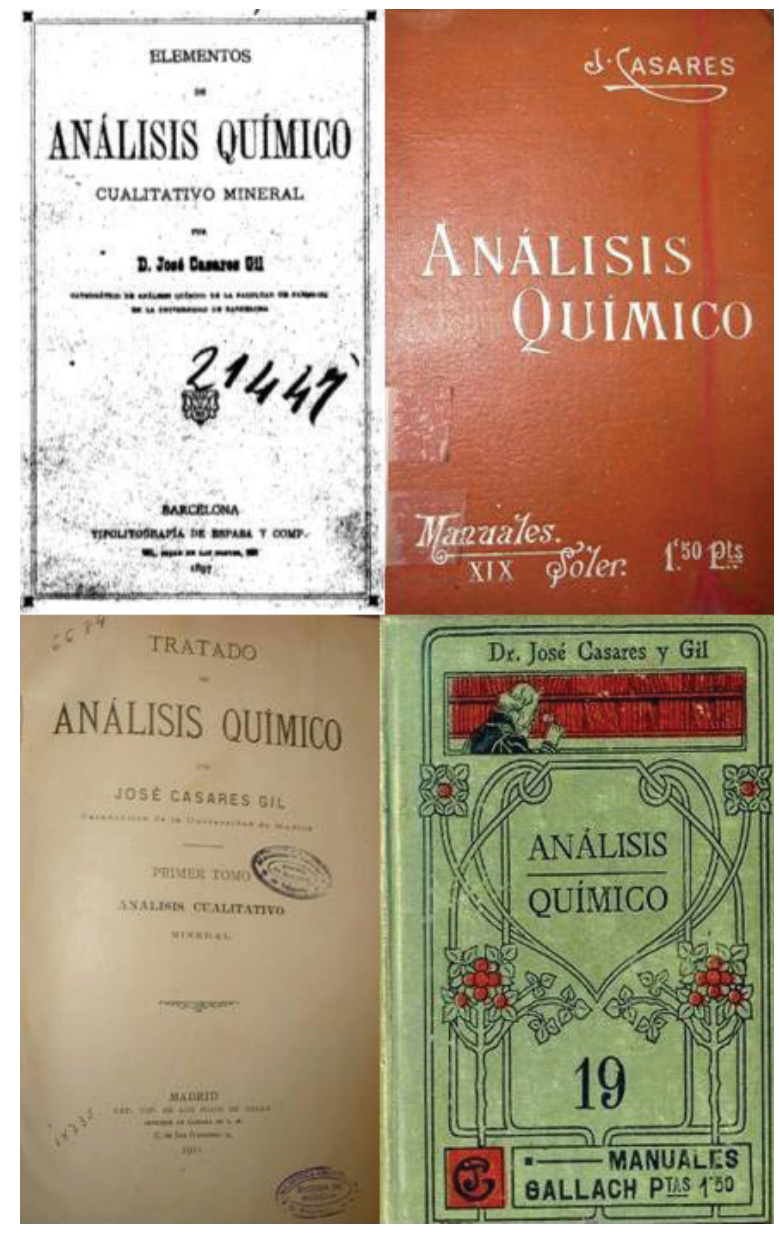

resultados de su primer viaje a Alemania fue la publicación de una obra titulada Elementos de análisis químico cualitativo mineral, editado en 1897 por la editorial Espasa pocos meses después de su regreso. Tanto su estancia en Alemania como la publicación del libro contribuyeron a reforzar el prestigio del autor. Por ejemplo, en un discurso en el que se daba la bienvenida a Casares como nuevo miembro de la Real Academia de Medicina de Barcelona en 1898 se enfatizó la relación entre ambas cuestiones, y se afirmó que el tratado de Casares permitiría propagar «las fructíferas y provechosas enseñanzas que había podido recibir de los sabios maestros de Múnich y Wiesbaden" y daría a conocer "los secretos de tecnicismo que había sabido arrancar a los más expertos y afamados químicos analíticos del mundo» (Codina, 1898). Más comedida fue la reseña publicada en la revista científica La Farmacia Española por el cate- 
drático de análisis químico de la facultad de ciencias de Barcelona Eugenio Mascareñas Hernández (18531934). Mascareñas destacó que Casares no se había limitado a recopilar y resumir obras extensas sino que le había dado su "sello propio y original» fruto del trabajo de laboratorio ${ }^{4}$. El propio prólogo del tratado de Casares también resaltaba esta cuestión al afirmar que todas las reacciones y todos los métodos habían sido "objeto de numerosos ensayos» previos (Casares, 1897). Además, Casares utilizó su prólogo para indicar que los públicos destinatarios del libro eran los alumnos de análisis químico de la facultad de farmacia a quienes recomendaba que lo utilizaran como "guía», así como para la "preparación de exámenes», pero también señaló que esperaba que su libro sirviera para despertar su «afición a los trabajos de laboratorio" (Casares, 1897). Es decir, el público destinatario de la obra incluía tanto a sus estudiantes de farmacia como a todos aquellos interesados en un compendio general de análisis químico.

La publicación de los Elementos de análisis químico de José Casares tuvo muy buena acogida entre los catedráticos y profesores españoles, que hasta entonces utilizaban, principalmente, traducciones de tratados alemanes. El éxito del libro de José Casares animó a la casa editorial a preparar una versión reducida que pudiera llegar a públicos más amplios. En 1905 y 1918, las casas editoriales Espasa y Calpe publicaron dos versiones más cortas (de unas 180 páginas) del Tratado de análisis químico de Casares, dentro de sus colecciones popularizadoras Soler y Gallach (Casares, 1905; Casares, 1918). Este tipo de obras, son un reflejo del surgimiento de nuevas audiencias para la química en la periferia europea, animadas por el interés de nuevos públicos como estudiantes de escuelas profesionales y una clase media cada vez más instruida. La elección de conocidos autores como Casares, no sólo muestra el interés de las editoriales por contar con algunos de los nombres más conocidos de la ciencia y la cultura española, sino que también refleja otra de las características de la periferia europea consistente en que las figuras más representativas debían esforzarse por justificar sus actividades científicas y buscar el apoyo de la sociedad (Nieto-Galán, 2004). Obras como estas, con precios asequibles y dirigidas a un público amplio, suponían una magnífica oportunidad para mostrar los beneficios que suponía la disciplina en la que trabajaban los científicos. En las primeras páginas del manual Soler, Casares afirmaba que el análisis químico no sólo era un "excelente método pedagógico» sino que también era el medio utilizado por los químicos para descubrir elementos, reconocer los minerales, buscar el arsénico en las vísceras de un cadáver, determinar la cantidad de alcohol de un vino y muchas otras aplicaciones interesantes o útiles. Es decir, publicaciones popularizadoras como la de José Casares, contribuyeron a definir al análisis químico como objeto de estudio y a identificar a su autor ante una audiencia muy amplia como un experto en esa materia.

El éxito de sus libros anteriores, la realización de nuevos viajes a Alemania en 1899 y 1905, así como su traslado como catedrático a la facultad de farmacia de Madrid (también en 1905), le impulsó a reformar y reeditar su obra. En 1911 realizó numerosas adiciones en su libro reorganizándolo, ampliándolo intensamente y cambiándole el título para denominarlo Tratado de análisis químico cualitativo mineral. Este libro supuso la confirmación definitiva de José Casares como autor de manuales de esta materia en España durante varias décadas, y la obra tuvo tan buena acogida que fue publicada nuevamente en diez ocasiones. Como se puede observar en la tabla 1, las primeras cuatro ediciones de este tratado académico (publicadas entre 1911 y 1935) corresponden a su etapa como catedrático en Madrid. Posteriormente, estando ya jubilado, aparecieron las ediciones quinta, sexta y séptima (en 1948, 1954 y 1956), que presentaban a José Casares como autor pero añadían que la obra estaba «continuada» por Román Casares López (1908-1990), su sobrino-nieto y sucesor en la cátedra de análisis químicos. Estas ediciones supusieron un importante cambio en la obra que implicó su organización en tres volúmenes con nuevos contenidos en bromatología. La incorporación de ayudantes y colaboradores en la cátedra de Madrid consolidó una nueva línea de trabajo centrada en los análisis de alimentos y reforzada en 1954 con la inauguración de la Escuela de Bromatología de la universidad de Madrid. A partir de entonces se mantuvo un volumen dedicado al análisis cualitativo mineral, otro al análisis cuantitativo general y, un tercero a los análisis químicos aplicados a los alimentos las aguas y los tóxicos. Después del fallecimiento de José Casares, el libro continuó publicándose, figurando únicamente el nombre de Román Casares como autor de las ediciones de 1966 y 1969 (octava y novena edición). Por último, la décima edición publicada entre 1975 y 1978, mostraba a Román Casares como autor principal y se añadió que la obra contaba «con la colaboración de los profesores» León Villanúa Fungairiño (1918-2004) y Pedro García Puertas (1925-1998), ambos doctores en farmacia y profesores de la facultad de farmacia de Madrid (Suay-Matallana, 2014b). La incorporación de nuevos capítulos vinculados a sus líneas de investigación más recientes 
implicó también que la extensión de la obra creciera considerablemente con el tiempo. Así las sucesivas ediciones aumentaron progresivamente desde las 151 páginas de la edición de 1897 hasta estabilizarse en torno a 1600 páginas a partir de la tercera edición de 1923, como puede comprobarse en la tabla 1.

Las nuevas ediciones también introdujeron otras mejoras como el incremento del número de índices, tablas y cuadros sinópticos. Estos elementos no sólo evitaban complejas descripciones y organizaban y secuenciaban los procedimientos sino que también aportaban detalles difíciles de expresar por escrito, como líneas espectrales, colores, precipitados, etc., que facilitaban el trabajo experimental (Tomic, 2005). La obra de Casares fue incorporando también nuevos grabados e ilustraciones que ofrecían una gran riqueza de detalles de mucha utilidad, e incluso añadió un apéndice dedicado a la legislación aplicable a las materias discutidas en el libro, lo cual es un ejemplo de su adaptación a los posibles usos de sus lectores así como del interés del autor por relacionarlo con su contexto local. Estos elementos reflejaban un culto por el detalle que resultaba fundamental en las obras dedicadas a la química analítica en la que se requería información muy detallada, tanto para guiar a los estudiantes como para describir los materiales e instrumentos y para tratar de evitar o prevenir los errores experimentales.

En el apartado siguiente se estudiarán las estrategias que siguió José Casares en su Tratado de análisis químico con la intención de institucionalizar la química analítica como disciplina en España.

\section{EL TRATADO DE ANÁLISIS QUÍMICO Y LA CONSTRUC- CIÓN DE UNA IDENTIDAD DISCIPLINAR}

Los libros de texto son un elemento crucial en el proceso de consolidación de una disciplina académica. La existencia de una disciplina académica no sólo comporta la existencia de una tradición pedagógica y de obras educativas, sino que suele implicar también un conjunto de problemas y valores compartidos, métodos y prácticas comunes para resolverlos, una literatura académica, un espacio institucional, cierto grado de reconocimiento externo y una genealogía de personajes y momentos fundadores (Golinski, 1998). Otros elementos que contribuyen a la consolidación de una disciplina académica son el incremento del número de plazas académicas bien remuneradas en instituciones públicas y privadas; el aumento de programas específicos como el doctorado; la expansión de prácticas en laboratorios que permitan a los estudiantes adquirir destrezas avanzadas; el aumento de publicaciones es-
Tabla 1. Evolución de las distintas ediciones del Tratado de análisis químico de José Casares

Título: Elementos de análisis químico cualitativo mineral. Año de publicación: 1897

División de la obra: 1 volumen, 185 pp.

Autoría: José Casares Gil.

Título: Análisis Químico.

Años de publicación: 1905

División de la obra: 1 volumen, 176 pp.

Autoría: José Casares Gil, Colecciones popularizadoras Soler

Título: Tratado de análisis químico, 1 ed.

Años de publicación: 1911-1913.

División de la obra: 2 volúmenes, 606 y 284 pp.

Autoría: José Casares Gil.

Título: Tratado de análisis químico, 2 ed.

Años de publicación: 1916-1921.

División de la obra: 2 volúmenes, 590 y 972 pp.

Autoría: José Casares Gil.

Título: Análisis Químico.

Año de publicación: 1918

División de la obra: 1 volumen, $180 \mathrm{pp}$.

Autoría: José Casares Gil, Colecciones popularizadoras Gallach.

Título: Tratado de análisis químico, 3 ed.

Años de publicación: 1923-1927.

División de la obra: 2 volúmenes, 622 y 999 pp.

Autoría: José Casares Gil.

Título: Tratado de análisis químico, 4 ed.

Años de publicación: 1933-1935.

División de la obra: 2 volúmenes, 673 y 973 pp.

Autoría: José Casares Gil.

Título: Tratado de análisis químico, 5 ed.

Años de publicación: 1948-1951.

División de la obra: 3 volúmenes, 482, 505 y 509 pp.

Autoría: José Casares Gil «continuada por R. Casares López».

Título: Tratado de análisis químico, 6 ed.

Año de publicación: 1954.

División de la obra: 3 volúmenes, 482, 482 y 509 pp. Autoría: José Casares Gil «continuada por R. Casares López».

Título: Tratado de análisis químico, 7 ed.

Años de publicación: 1956-1958.

División de la obra: 3 volúmenes, 482, 530 y 585 p. Autoría: José Casares Gil «continuada por R. Casares López».

Título: Tratado de análisis químico, 8 ed.

Años de publicación: 1966-1967

División de la obra: 3 volúmenes, 482, 531 y 576 p. Autoría: Román Casares López «catedrático de la Universidad de Madrid».

Título: Tratado de análisis químico, 9 ed.

Años de publicación: 1969-1973.

División de la obra: 3 volúmenes, 423, 507 y 513 pp.

Autoría: Román Casares López «catedrático de la Universidad de Madrid».

Título: Tratado de análisis químico, 10 ed.

Años de publicación: 1975-1978.

División de la obra: 3 volúmenes, 440, 507 y 513 pp.

Autoría: «Por el catedrático R. Casares López, con la colaboración

de los profesores L. Villanúa Fungairiño y P. García Puertas». 
pecializadas y estudios monográficos; el crecimiento de instituciones científicas asociadas con el cultivo de la disciplina; la creación de un sistema más o menos autónomo de recompensa para científicos en las propias instituciones; y la progresiva diferenciación entre especialistas profesionales y amateurs (lo que también puede implicar la exclusión de determinados grupos de científicos) (Jo Nye, 1996). Sin embargo, el esquema de Jo Nye - utilizado preferente para analizar un momento histórico concreto- puede ser enriquecido por otros trabajos más extensos que tengan en cuenta como diferentes generaciones de científicos de un área utilizan distintos elementos para definir o limitar sus fronteras disciplinares (Gavroglu y Simões, 2012). Además, otros autores también discuten las relaciones existentes entre disciplinas, comunidades científicas y profesionalización de la ciencia, y han definido las disciplinas como un sistema autónomo con vínculos particulares con el mundo extra-científico (técnica, industria, política, enseñanza, etc.), que permitían a sus integrantes obtener recursos e interesar así al público (Stichweh, 1994). Desde la historia de la educación también se ha subrayado la constante presión que los profesores, alumnos, gobiernos e instituciones ejercen sobre las disciplinas escolares (Chervel, 1988; Viñao, 2006). En este sentido, también se ha discutido como el desarrollo de disciplinas científicas está estrechamente relacionado con la combinación de prácticas docentes y de investigación (Simon, 2011). A continuación se discutirá el papel de Casares y su tratado en la creación de una identidad disciplinar para la química en España haciendo uso del esquema propuesto por la historiadora de la ciencia estadounidense Mary Jo Nye que identificó los siguientes seis elementos fundamentales: una genealogía definida, una literatura básica, un conjunto de prácticas y rituales, un espacio propio, reconocimiento externo y valores compartidos (Jo Nye, 1993).

El primer rasgo constitutivo señalado por Jo Nye (1993) implica la definición de una "genealogía» que establezca una memoria compartida de la disciplina: con sus héroes fundadores, sus momentos cruciales y sus grandes descubrimientos. Al igual que también apuntó en su día Thomas S. Kuhn (1922-1996), estos recursos históricos ayudan a dar una imagen de continuidad de teorías y prácticas químicas, oscureciendo las posibles revoluciones del pasado, contribuyendo así a la construcción de la autoridad disciplinar. Casares hizo uso de estos elementos históricos al incluir en su Tratado de análisis químico múltiples referencias a personajes y pasajes heroicos de la química, algunos de ellos de forma muy explícita. Por ejem- plo, el apartado dedicado a la teoría de las soluciones comienza con un "resumen histórico» en el que se hacía referencia a las "célebres investigaciones» cinéticas y termoquímicas propuestas por Jacobus Henricus van 't Hoff (1852-1911) y se mencionan los trabajos de famosos cultivadores de la ciencia como Robert Boyle (1627-1691), Joseph Louis Gay-Lussac (1778-1850), Svante Arrhenius (1859-1927) o Michael Faraday (1791-1867) (Casares, 1911). En otro apartado, en el que introdujeron recomendaciones experimentales, Casares incluyó varios párrafos del Dictionnaire de Chymie - publicado en 1766 por el químico francés Pierre J. Macquer (1718-1784)- y afirmó que «pese al tiempo transcurrido resumía admirablemente las reglas que el analista debe tener presentes en su trabajo» (Casares, 1911). Además, en la introducción también se mencionan los trabajos realizados por otros químicos como Frederick P. Treadwell (1857-1918), Wilhelm C. Böttger (18711949), Alexander Classen (1843-1934), William Crookes (1832-1919), Frank Clowes (1848-1923) y Georg P. Max Dittrich (1864-1913). De esta forma, Casares mostraba no sólo un amplio dominio de la literatura química internacional (fundamentalmente alemana) sino que contribuía a dotar a la química analítica de un pasado identitario.

El segundo elemento señalado por Jo Nye (1993) es la existencia de una literatura propia y características, que incluye obras de referencia básicas que identifican la disciplina y definen su lenguaje. En el caso del tratado estudiado, Casares reconoció el valor de esta literatura al afirmar en su prólogo que «la conocida obra de Fresenius» había sido su guía principal (Casares, 1911). En otra publicación de Casares dedicada a los análisis de aguas el reconocimiento a ese autor fue incluso mayor y llegó a afirmar que los trabajos analíticos de Fresenius eran una «obra clásica que sirve de guía» y que "con pocas modificaciones, sirve todavía de base en la mayor parte de las investigaciones de este género" (Casares, 1909). Sin dura Carl Remigius Fressenius (1818-1897) fue un personaje crucial en la historia de la química analítica y él mismo formó parte de una saga familiar dedicada a esta disciplina que incluso editó durante varias generaciones la prestigiosa revista Zeitschrift für Analytische Chemie (denominada desde 1990 Analytical and Bioanalytical Chemistry). Sin embargo, son necesarios más trabajos comparados para conocer si las genealogías familiares juegan un mismo papel en una disciplina o en una especialidad, considerada esta última como parte de un cuerpo disciplinar más amplio 5 . 
La existencia de prácticas y rituales codificados son el tercer rasgo identificador de disciplinas científicas señalado por Jo Nye (1993). Estos elementos incluyen tanto las prácticas relacionadas con el trabajo de laboratorio - un espacio de aprendizaje experimental y de adquisición de conocimiento tácito- como las prácticas docentes, académicas y conmemorativas $^{6}$. Los primeros capítulos del Tratado de análisis químico de José Casares, son un ejemplo del esfuerzo del autor para lograr que los lectores se "familiarizasen con los procedimientos descritos» por lo que dedicó las primeras setenta páginas del libro a explicar cuestiones prácticas como la organización de un laboratorio, la descripción de los sistemas de alumbrado y calefacción, la explicación de cómo trabajar el vidrio de laboratorio o cómo realizar numerosas operaciones químicas, así como la descripción de la mejor forma de realizar ensayos y de preparar y seleccionar los reactivos más apropiados. Por otra parte, las actividades conmemorativas y la asistencia a seminarios, congresos e incluso homenajes y conmemoraciones también han sido consideradas como «rituales» que ayudaban a recrear tradiciones y legitimar la disciplina. Casares reivindicó la importancia del análisis químico en los diferentes congresos a los que asistió, como los organizados por la Asociación Española para el Progreso de las Ciencias - de la que formó parte y en el que se encargó del discurso inaugural de la sección de físico-química en 1911- o bien en el IX Congreso de la Unión Internacional de Química Pura y Aplicada celebrado en 1934 en Madrid, en el que fue nombrado vocal y que significó un importante impulso internacional para los químicos analistas y farmacéuticos españoles. Bastantes años después, con motivo de la celebración de un congreso sobre espectrometría celebrado en Madrid en 1969, los organizadores recordaron el nombre de Casares para subrayar que la ciencia española tenía una "ejecutoria muy destacada» en el manejo de esta técnica química?.

El cuarto rasgo indispensable en la creación de identidades disciplinares señalado por Jo Nye (1993) hace referencia a la existencia de espacios propios, academias y sociedades científicas. Casares comenzó su tratado con un capítulo dedicado precisamente a los espacios en el que ofrecía sus recomendaciones personales para configurar un laboratorio sencillo en el que la mesa de trabajo era uno de los elementos fundamentales y también recomendaba los materiales necesarios para los análisis más básicos. Sus viajes al extranjero así como la dirección del laboratorio de su facultad y el laboratorio de aduanas (cuya reorganización dirigió en la década de 1920) le proporcionaron una notable experiencia en la gestión de estos espacios. Por otra parte, Casares participó activamente en las actividades organizadas por diversas sociedades científicas creadas en España a principios del siglo XX. Entre 1935 y 1957 presidió la reorganizada Academia Nacional de Farmacia (creada en 1932), así como la Real Academia de Ciencias de Madrid (entre 1940 y 1958), y también presidió en 1911 la Sociedad Española de Física y Química (fundada en 1903). Esta última convirtió su revista Anales de Física y Química, en un prestigioso medio de comunicación para los científicos españoles y que varias décadas después se convertiría en una de las diez revistas mundiales más importantes en el campo de la química analítica (Boig y Howerton, 1952).

El quinto elemento considerado por Mary Jo Nye (1993) implica la existencia de reconocimiento externo tanto por los miembros de la propia disciplina como por otros grupos. En este sentido, la contribución de José Casares fue muy relevante debido, en parte, a su proyección política y a su participación como miembro de otras instituciones y comisiones institucionales, a su nombramiento como director del laboratorio de aduanas, a los encargos privados que recibió para analizar diversas aguas minerales y a la recepción de numerosos homenajes y premios, por ejemplo, los doctorados honoris causa por la universidad de Múnich y de Oporto o el premio de química de la fundación Juan March otorgado como reconocimiento a sus mediaciones internacionales y su trayectoria académica ${ }^{8}$. En 1947, Fernando Burriel Martí (1905-1978), que era catedrático de análisis químico de la facultad de ciencias de Madrid, mostró su reconocimiento hacia José Casares afirmando que podía ser considerado «nuestro padre de la química analítica en España» (Burriel, 1947) ${ }^{9}$. Otros testimonios similares, publicados en diccionarios históricos de la ciencia también destacan la importancia pedagógica del tratado de Casares y su contribución para la «formación en análisis químico de la mayoría de químicos españoles» contemporáneos (Bermejo y Fandiño, 2003). Es decir, los testimonios anteriores vinculan el impacto del libro y el prestigio del autor con su reconocimiento como destacado protagonista de la consolidación de esta disciplina en las aulas españolas.

Finalmente, el último rasgo señalado por Jo Nye (1993) para analizar la cohesión y la identidad de una disciplina implica el reconocimiento de valores compartidos y problemas aún no resueltos por la comunidad académica. Como se ha mostrado anteriormente, las colecciones popularizadoras editadas por 
Soler y Gallach son un ejemplo del interés de Casares por ampliar los públicos interesados en los análisis químicos convenciéndoles de la utilidad de la analítica y de sus variadas aplicaciones. En este sentido, Casares compendió que podía reforzar la publicidad de su disciplina si era capaz de relacionarla con las necesidades locales y la adaptaba a los intereses de sus nuevos lectores. Por ello, Casares recordó en el prólogo de sus Elementos de análisis químico que el análisis no sólo tenía «la mayor importancia en química» sino que constituía "el método de investigación y es la mejor escuela para adquirir la sagacidad, la paciencia y la constancia necesarias en las ciencias experimentales» enfatizando además que tenía la «ventaja de no exigir el empleo de aparatos costosos ni complicados» (Casares, 1897).

José Casares utilizó su libro y su autoridad científica como experto analista para mitificar, establecer patrones de aprendizaje, reglas y costumbres, así como para institucionalizar espacios y crear sociedades que identificaran los ideales y valores disciplinares compartidos. Posiblemente el esquema propuesto por Jo Nye podría enriquecerse con las aportaciones propuestas por otros autores -como Simon, Gavroglu y Simões - para definir con más detalle las identidades y los límites entre especialidades y disciplinas científicas (Simon, Gavroglu y Simões, 2012). En todo caso, los elementos discutidos muestran como las actividades de José Casares junto con su Tratado de análisis químico publicado por Casares contribuyó a consolidar la química analítica en España en el primer tercio del siglo XX.

\section{CONCLUSIÓN}

Los apartados anteriores se han centrado en analizar cómo las múltiples ediciones Tratado de análisis químico publicado por José Casares contribuyeron a construir una identidad disciplinar en esa materia. Los libros de texto son herramientas didácticas que se configuran como resultado de un proceso complejo en el que participaron un gran número de protagonistas. Además, los libros de texto fueron importantes fuentes de legitimidad para sus autores y les permitieron convertirse en voces autorizadas para abordar diversos temas relacionados con la química, el análisis químico y sus aplicaciones. En el caso de José Casares, su tratado no sólo reforzó su autoridad científica y su consideración como experto sino que tuvo además un papel crucial en la consolidación e institucionalización del análisis químico como disciplina en las universidades españolas a principios del siglo XX.
José Casares realizó un esfuerzo por consolidar una especialidad académica nueva (la química analítica) apropiando y adaptando en sus numerosos textos las enseñanzas recibidas durante sus viajes. La buena recepción de su tratado se vio favorecida por el nuevo clima renovador de la enseñanza experimental y científica, y recibió una cálida acogida por parte de otros profesores y colegas de la universidad. A pesar de que era consciente de las limitaciones del sistema científico español, José Casares trató de modernizar las prácticas experimentales. El éxito de los Elementos de análisis químico, entre los estudiantes universitarios, se vio reforzado por la publicación de unas pequeñas obras de popularización que le permitieron acceder a nuevos sectores de la población interesados por cuestiones científicas. Con las ediciones popularizadoras José Casares se dirigía a unos públicos destinatarios más amplios -no limitados a las aulas universitarias - fomentando los usos de su libro más allá de las universidades y reforzando así su papel como experto y promocionando la química analítica en diversas esferas. Las versiones previas del tratado publicado por Casares le sirvieron como ejemplo para la redacción de las ediciones posteriores. Algunos de los cambios realizados en el tratado muestran los esfuerzos del autor por ajustarse a los cambios legislativos, y por incluir una gran riqueza de detalles (como tablas, cuadros o apéndices) para organizar la materia y secuenciar los conocimientos.

El Tratado de análisis químico contribuyó a consolidar la química analítica en España a través de la creación de rasgos característicos de una identidad disciplinar: la identificación de personajes heroicos, la formulación de instrumentos y espacios icónicos - la formulación de problemas analíticos según un modo pautado y definido. De este modo, este tratado sirvió para que José Casares fuera reivindicado por la generación de químicos que le sucedió como uno de los padres fundadores de la química analítica en España y una autoridad académica de referencia en dicha disciplina. El sintético esquema propuesto por Jo Nye desarrollado en este artículo puede dar lugar a trabajos más extensos en los que se estudien los procesos de consolidación de una disciplina considerando diferentes generaciones de científicos. En el caso español, un estudio cronológicamente más amplio podría ofrecer nuevos detalles sobre la evolución disciplinar de la química analítica desde los análisis minerales, vegetales, de aguas o los ensayos de metales (docimasia) realizados en el siglo XIX, con la renovación de la química y la farmacia durante el primer tercio del siglo $\mathrm{XX}$ o los trabajos realizados 
por la generación de científicos posterior a la guerra civil. Las reseñas de compañeros de Casares, como Codina y Mascareñas, destacaron el esfuerzo realizado por Casares para adaptar esta disciplina a su contexto local y ayudaron a afianzar el éxito del libro y el prestigio de su autor, mientras que los comentarios elogiosos de químicos posteriores a Casares, como Buriel y Bermejo, reivindicaron su figura para crear una conciencia histórica local en torno a la química analítica. En todo caso, el esquema de discusión propuesto por Jo Nye ha permitido evaluar la contribución de José Casares a la institucionalización y al refuerzo de la identidad disciplinar del análisis químico en España. Por todo ello, el Tratado de análisis

\section{NOTAS}

1. Sobre la regulación de los libros de texto de secundaria véase: (Shapiro, 2012).

2. Una versión previa de este artículo fue presentada en el congreso $9^{\text {th }}$ International Conference for the History of Chemistry, Chemistry in Material Culture (Uppsala, 2013) y en la tesis doctoral que titulé Análisis químico y expertos en la España contemporánea: Antonio Casares Rodríguez (1812-1888) y José Casares Gil (1866-1961) (Universidad de Valencia, 2014).

3. Una publicación - realizada en un marco conmemorativo por un catedrático de química analítica- con abundante información sobre históricos químicos analíticos españoles es: (Arribas, 1985).

4. Mascareñas, E. (1897), Variedades, Apuntes bibliográficos, La Farmacia Española, 11, pp. 167-168.

5. Para una discusión más detallada -centrada en el ámbito médico- sobre disciplinas y especialidades ver: (Weisz, 2006).

\section{BIBLIOGRAFÍA}

Arribas Jimeno, S. (1985), Introducción a la Historia de la Química Analítica en España, Oviedo, Universidad de Oviedo, p.33.

Barona Vilar, J.L. (2007), "Los laboratorios de la Junta para Ampliación de Estudios e Investigaciones Científicas (J.A.E.) y la Residencia de Estudiantes (1912-1939)", Asclepio, 59 (2), pp. 87-114.

Bensaude-Vincent, B. (2007), "College Chemistry: how a textbook can reveal the values embedded in chemistry", Endeavour, 311 (4), pp. 140-144.

Bermejo Patiño, M. y Fandiño Veiga, X.R. (2003), “José Casares Gil",Álbum da Ciencia, [en línea], disponible en: http://www.culturagalega.org/albumdaciencia/detalle. php?id=454\&autor=Jos\%E9\%20Casares\%20Gil, [consultado el $25 / 10 / 2015]$. químico así como la autoridad académica y el poder institucional adquirido por José Casares contribuyeron a que esa materia se consolidara y cohesionara como disciplina en las universidades españolas durante la primera mitad del siglo XX.

\section{AGRADECIMIENTOS}

Este artículo ha sido posible gracias al proyecto español de investigación "Ciencia, medicina y ley en España (1845-1940)" (HAR2012-36204-C02-01/HIST), así como al proyecto de investigación portugués "Experts, Institutions and Globalisation (EIG)" (UID/ HIS/00286/2013).

6. Sobre esta cuestión ver también: (Bensaude-Vincent, 2007).

7. Hoy comienza en Madrid el XV Congreso Internacional de Espectroquímica, (1969), Hoja del Lunes, 18, (26/05/1969).

8. Esta fundación fue creada por el banquero afecto al régimen franquista Juan March Ordinas (1880-1962). En marzo de 1956 José Casares recibió el premio de química de la Fundación Juan March "que corona toda una vida dedicada a la química" dotado con la considerable cantidad de 500000 pesetas. Archivo General de la Universidad Complutense de Madrid: 134/10-25, no 565; Leg. 33; Expte. 1,1.

9. Fernando Burriel escribió (junto con Felipe Lucena Conde y Siro Arribas Jimeno) el libro titulado Química analítica cualitativa. Esta obra fue, posiblemente, el libro de química analítica más utilizado en España en la etapa posterior a la de Casares Gil y cuenta con dieciocho ediciones publicadas entre 1952 y 2008.

Bertomeu Sánchez, J.R. y García Belmar, A. (eds.) (2002), Abriendo las cajas negras: Los instrumentos científicos de la Universidad de Valencia, Valencia, Universitat de València.

Bertomeu Sánchez, J.R.; García Belmar, A.; Lundgren, A. and Pationitis, M. (2006), "Introduction: Scientific and Technological Textbooks in the European Periphery", Science \& Education, 15, pp. 657-665, p. 657.

Boig, F.S. and Howerton, P. W. (1952), "History and Development of Chemical Periodicals in the Field of Analytical Chemistry: 1877-1950", Science, 115 (2995), pp. 555-560, p. 560.

Burriel, F. (1947), "Nota biográfica del Excmo. Sr. D. José Casares Gil”, Anales de Física y Química, 43, pp. 801-826.

Casares Gil, J. (1897), Elementos de análisis químico cualitativo mineral, Barcelona, Espasa. 
Casares Gil, J. (1905), Análisis químico: (tratado elemental, Barcelona, Manuel Soler.

Casares Gil, J (1909), Consideraciones acerca de algunos métodos empleados en el análisis de aguas minerales, Madrid, Est. Tipográfico Pontejos.

Casares Gil, J (1911), Tratado de análisis químico, Madrid, Imp. Viuda e Hijos de Tello.

Casares Gil, J. (1918), Análisis químico: (tratado elemental), Manuales-Gallach no 19, Barcelona, Calpe.

Chervel, A. (1988), “L’histoire des disciplines scolaires: réflexions sur un domaine de recherches", Histoire de l'éducation, 38, pp. 59-121.

Codina Langlín, R. (1898), "Discurso de contestación". En: Casares Gil, J. Fundamentos que sirven de base a las fórmulas de estructura y de la importancia de las mismas en la biología, Barcelona, Real Academia de Medicina y Cirugía de Barcelona, pp. 33-62, p. 37

Darnton, R. (2007), "'What is the history of books?' revisited", Modern Intellectual History, 4 (3), pp. 495-508.

García Belmar, A. y Bertomeu Sánchez, J.R. (2001), "Viajes a Francia para el estudio de la química, 1770-1833", Asclepio, 53 (1), pp. 95-139, p. 97

Golinski, J. (1998), Making natural knowledge: constructivism and the history of science, Chicago, University of Chicago Press, pp. 68-69.

González de Posada, F. (2005), “Enrique Moles Ormella (18831953) Farmacéutico, químico y artista”, Anales Real Academia Nacional de Farmacia, 71, pp. 673-702, p. 693.

Jo Nye, M. (1993), From chemical philosophy to theoretical chemistry: dynamics of matter and dynamics of disciplines, 1800-1950, Berkeley, University of California Press, p. 19.

Jo Nye, M. (1996), Before big science: the pursuit of modern chemistry and physics 1800-1940, Cambridge, Harvard University Press.

Kaiser, D. (ed.) (2005), Pedagogy and the Practice of Science: Historical and Contemporary Perspectives, Boston, MIT.

Gavroglu, K. and Simões, A. (2012), Neither Physics nor Chemistry: A History of Quantum Chemistry, Cambridge, Massachusetts Institute of Technology, pp. 259-261.

Nieto-Galán, A. (2004), "Free radicals in the European periphery: 'translating' organic chemistry from Zurich to Barcelona in the early twentieth century", British Journal for the History of Science, 37 (2), pp. 167-191.

Portela, E. (1983), "Casares Gil, José". En: López Piñero, J.M. et al. Diccionario Histórico de la Ciencia Moderna en España, v. 1, Barcelona, Ed. Península, pp. 188-190.

Roldán Guerrero, R. (1975), Diccionario biográfico y bibliográfico de autores farmacéuticos españoles, v. 1. Madrid, Imp. de P.H.O.E, pp. 599-604.

Shapiro, A.R. (2012), "Training and Popularization: Regulating Science Textbooks in Secondary Education" Isis, 103 (1), pp. 99-110.

Simon, J. (2011), Communicating Physics: The Production, Circulation and Appropriation of Ganot's Textbooks in France and England (1851-1887), London, Pickering and Chatto, pp. 213-214.

Stichweh, R. (1994), "La structuration des disciplines dans les universités allemandes au XIXe siècle", Histoire de l'éducation, 62, pp. 55-73, p.56.

Suay-Matallana, I. (2014a), "La colaboración científica y los espacios de la química: un estudio de caso español en la primera mitad del siglo XX", Revista Española de Documentación Científica, 37 (4), pp. 01-11.

Suay-Matallana, I. (2014b), Análisis químico y expertos en la España contemporánea: Antonio Casares Rodríguez (18121888) y José Casares Gil (1866-1961), Valencia, Universidad de Valencia, p. 172.

Szabadvary, F. (1966), History of analytical chemistry, Oxford, Pergamon Press, p. 150.

Tomic, S. (2005), "Transmettre le savoir-faire: les cours d'analyse chimique au 19e siècle, Le cours magistral: modalités et usages (XVle-XXe siècles)", [en línea], disponible en: http:// www.inrp.fr/she/cours_magistral/expose_analyse_chimique/expose_analyse_chimique_complet.htm, [consultado el 25/10/2015].

Vicedo, M. (2012), "The secret lives of textbooks", Isis, 103 (1), pp. 83-87, p.87.

Viñao, A. (2006), "La historia de las disciplinas escolares", Historia de la Educación, 25, pp. 225-242.

Weisz, G. (2006), Divide and conquer: a comparative history of medical specialization, Oxford, Oxford University Press. 